\title{
2017. Signal intensity dynamics of malignant lymphoma in diffusion-weighted magnetic resonance imaging compared to positron emission tomography
}

\author{
Rytis Bliūdžius ${ }^{1}$, Laima Dobrovolskienè ${ }^{2}$, Algidas Basevičius ${ }^{3}$, Vincentas Veikutis ${ }^{4}$, \\ Matas Juškevičius ${ }^{\mathbf{5}}$, Vaidas Oberauskas ${ }^{6}$ \\ ${ }^{1,5}$ Medical Academy, Lithuanian University of Health Sciences, \\ Mickevičiaus 9, LT-44307, Kaunas, Lithuania \\ ${ }^{2,3}$ Department of Radiology, Lithuanian University of Health Sciences, \\ Mickevičiaus 9, LT-50009, Kaunas, Lithuania \\ ${ }^{4}$ Institute of Cardiology, Lithuanian University of Health Sciences, \\ Sukilèlių 15, LT-50009, Kaunas, Lithuania \\ ${ }^{6}$ Lithuanian University of Health Sciences, Mickevičiaus 9, LT-44307, Kaunas, Lithuania \\ ${ }^{1}$ Corresponding author \\ E-mail: ${ }^{1}$ rytisbliudzius@gmail.com, ${ }^{2}$ laimadobra@gmail.com, ${ }^{3}$ algidas.basevicius@kaunoklinikos.lt, \\ 4vincentas.veikutis@lsmuni.lt, ${ }^{5}$ matasjusk@gmail.com, ${ }^{6}$ vaidas.oberauskas@lsmuni.lt \\ Received 10 March 2016; received in revised form 4 May 2016; accepted 16 May 2016 \\ DOI http://dx.doi.org/10.21595/jve.2016.17177
}

\begin{abstract}
This article discusses a study performed with an aim to determine the usefulness of diffusion-weighted MRI in pre-treatment assessment and evaluation of chemotherapy response of mediastinal lesions of malignant lymphoma by analysing signal intensity, accumulation of contrast agent and changes in values of ADC. Comparing the results of PET/CT and DW-MRI was another goal of this study. We reviewed data of 21 patients that underwent treatment during years 2013-2014 at the Hospital of Lithuanian University of Health Sciences Kaunas Clinics and had DW-MRI and PET/CT scans carried out. For all patients the diagnosis of malignant lymphoma in mediastinal lymph nodes was confirmed histological. Whether the lymphoma was in active state was determined by PET/CT or histological data. In MRI scans, signal intensity and ADC values were evaluated, while in PET/CT maximum and average standardized uptake values were assessed. Patients underwent chemotherapy and follow up scans were performed to evaluate response. In total, $25 \mathrm{PET} / \mathrm{CT}$ and $50 \mathrm{DW}-\mathrm{MRI}$ scans were analysed. We found statistically significant difference of values of ADC in active and inactive states of malignant lymphoma of mediastinal lymph nodes. In follow up tests, performed during 2 years after treatment, a tendency of decreasing ADC values over time was seen when chemotherapy was successful and no relapse was observed. Among the reviewed cases, DW-MRI had slightly worse specificity than PET/CT in differentiation of malignant and benign changes in lymph nodes.
\end{abstract}

Keywords: malignant lymphoma, diffusion-weighted magnetic resonance imaging (DW-MRI), apparent diffusion coefficient (ADC), positron emission tomography/computed tomography (PET/CT).

\section{Introduction}

Lymphomas are a group of lymph proliferative diseases, classified into two types - Hodgkin's and non-Hodgkin's lymphomas. Morbidity for this illness seems to be rising lately, as about 450 new cases of lymphoma are found in Lithuania yearly [1]. Hodgkin's lymphomas are usually diagnosed for people at the age of 20-30 years, meaning it's a disease of young, active and working age people. Fortunately, malignant lymphomas show great response to chemotherapy, although the treatment has harsh side effects and oftentimes can be hard for patients to tolerate. Finding an optimal treatment strategy is one of the main goals when assessing lymphomas. Employing radiologic methods for correct primary staging of tumour and evaluation of response to treatment allows optimising the use of chemotherapy. Early assessment of the effect of chemotherapy means that treatment tactics can be changed or corrected on an early stage of disease, if seen not to be 
2017. SIGNAL INTENSITY DYNAMICS OF MALIGNANT LYMPHOMA IN DIFFUSION-WEIGHTED MAGNETIC RESONANCE IMAGING...

effective enough, while minimizing toxicity and expenses of ineffective chemotherapy and maximising the probability of successful outcomes [2].

For a long time computed tomography (CT) scans were used as the main method for assessment of lymphomas. Lately positron emission tomography combined with computed tomography (PET/CT) was tried to use more and more often and now became the standard for imaging lymphomas. The main drawback of PET could be lack of detailed anatomical information, while CT alone doesn't account for the clear functional changes in tissues. Fusing these images eliminates these drawbacks, allowing combining anatomical information of CT with functional information of PET in addition to better signal attenuation [3], thus improving both sensitivity and specificity of the scans. However, PET/CT is a costly, relatively slow sophisticated imaging technique and, most importantly, exposes the patient to marked ionizing radiation, so, as new imaging sequences are developed, diffusion-weighted magnetic resonance imaging (DW-MRI) could becoming a viable alternative to PET/CT. Its usefulness is apparent when scanning children, pregnant women or multiple follow-up tests have to be performed after treatment, as minimizing the amount of ionizing radiation gains even greater importance [2]. Also DW-MRI can be successfully used for patients allergic to iodine based contrast agent which is a common problem performing CT.

For the last few years, the use of MRI and DW-MRI for imaging tumours of lungs and mediastinum is becoming more frequent in Lithuania. MRI, combined with diffusion-weighted imaging can be used to assess both the morphology and functional activity of mediastinal tissue. One of significant advantages of DW-MRI could be high lesion to background tissue contrast, which allows better accuracy in determining the margins of lesion [4]. Higher soft-tissue contrast and higher spatial resolution compared to PET/CT makes false positive and false negative results less likely [5]. Prime cause of it is that MRI diffusion is based on the difference of movement of water molecules in biological tissues. Malignant tissues tend to have hyper cellular structure with enlarged hyper chromatic nuclei and high levels of macromolecular proteins. All these factors lead to a reduction of diffusion space for water molecules, resulting in the decrease of apparent diffusion coefficient (ADC) values. However, in necrotic tissue, breakdown of diffusion barriers facilitates the movement of water molecules, thus increasing the value of ADC [2]. Chemotherapy treatment also alters the structure of tumour tissue, leading to changes of ADC, what suggests DW-MRI is valuable in evaluation of treatment response or tumour relapse. Based on modern literature findings, DW-MRI could be a valid alternative to PET/CT in oncology, although further analysis is needed for specific tumour types such as lymphomas [5].

This study aimed to compare the diagnostic value of DW-MRI and PET/CT and specify whether it can be used as an alternative in diagnostics of mediastinal lesions of lymphomas.

\section{Materials and methods}

This retrospective study was performed at the Lithuanian University of Health Sciences Clinical Hospital, Department of Radiology. Clinical data and radiologic findings of 21 patients were reviewed. All of the patients had previously diagnosed lymphoma of mediastinal lymph nodes and underwent treatment during period of 2013-2014 years. DW-MRI and PET/CT scans were carried out for every patient. Diagnosis of malignant lymphoma was confirmed in accordance to histological findings for all patients. The patient group consisted of 12 women and 9 men, age varied from 21 to 78 years ( $39 \pm 15.6$ years average) whether the lymphoma was in an active state and was determined by PET/CT or histological data. MRI scans were performed with paramagnetic contrast agent using 1.5T Siemens Magnetom Avanto scanner, T1, T2, DWI sequences were carried out, using b values of 50, 400 and $800 \mathrm{~s} / \mathrm{mm}^{2}$ for diffusion images [18]. F-FDG radiopharmaceutical was used for PET/CT scans. In MRI scans, signal intensity and ADC values were evaluated, while maximum and average standardized uptake values were assessed in PET/CT scans. Patient underwent from 4 to 8 courses of chemotherapy, follow-up DW-MRI and PET/CT scans were performed to evaluate response when all courses were finished. 25 PET/CT 
and 50 DW-MRI scans were analysed. 20 patients had PET/CT and DW-MRI performed at the same stage of disease, the findings of which were compared considering histological data when findings were different between the two modalities. Lymphoma tissue was considered inactive if the masses disappeared or transformed into fibrous tissue.

Data was analysed using IBM SPSS statistics packet22.0. We used independent samples $T$ test and Spearman correlation coefficient, data was considered statistically significant, when $p \leq 0.05$.

\section{Results}

Each of 21 patients had histological confirmed malignant lymphoma. Hodgkin's lymphoma was diagnosed for 18 (85.7\%) patients, while non-Hodgkin's lymphoma- for $3(14.3 \%)$ of patients. The average age of patients with Hodgkin's lymphoma was lower, averaging 35 (SD 9.64) years, while non-Hodgkin's lymphoma's average was 65 (SD - 21.65) years. $14.3 \%$ $(n=3)$ of lymphomas were of stage I, $42.9 \%(n=9)$ - stage II, $23.8 \%(n=5)$ - stage III, $19 \%$ $(n=4)$ - stage IV. The locations of lesions (sometimes a few locations on the same patient) were founded as follows: sternal notch nodes $-90.5 \%(n=19)$, paratracheal $-76.2 \%(n=16)$, para-aortic $-47.6 \%(n=10)$, hilar $-33.3 \%(n=7)$, bifurcational $-23.8 \%(n=5)$ and paracardial $9.5 \%(n=2)$ of cases.

The average ADC value of active lymphoma was $1.131 \times 10^{-3}\left(\mathrm{SD}=0.41 \times 10^{-3}\right) \mathrm{mm}^{2} / \mathrm{s}$, while the average ADC value of inactive lymphoma was $0.391 \times 10^{-3}\left(\mathrm{SD}=0.552 \times 10^{-3}\right) \mathrm{mm}^{2} / \mathrm{s}$. The difference in ADC was statistically significant $(p<0.01)$. Calcification of lymph nodes made measuring average values of ADC difficult, causing high dispersion. In cases when successful outcome of treatment was known (lymphoma masses were inactive and no relapse was observed), follow-up scans performed in 2 years after treatment were analysed. Inverse correlation between time after treatment and value of ADC was seen $(\rho=-0.527, p=0.027)-$ values of ADC decreased as more time passed between follow up tests and final course of chemotherapy.

The results of DW-MRI and PET/CT carried out at the same stage of disease matched in 20 cases $(95.24 \%)$, where tumour characteristics such as maximum and average standardised uptake value (SUVmax, SUVavg) in PET/CT and ADC values in DW-MRI both suggested active or inactive state of lymphoma (Figs. 1, 2, 3), however in one case $(4.76 \%)$, DW-MRI could not reliably determine whether the tissue was malignant or not due to inflammatory reaction. A PET/CT scan had to be performed, which proved to be more specific, showing inactive state of lymphoma.

\section{Discussion}

In our cohort, diffusion-weighted MRI proved to be a reliable method for determining between active and inactive states of lymphoma, allowing evaluating both morphological and functional parameters of tumour. However, in a small amount of cases, MRI lacked enough specificity to differentiate between malignant tissue and changes in functional activity due to inflammation, and was requiring a simultaneous PET/CT scan. Every so often the post-treatment inflammatory process and particularly macrophage proliferation may cause false-positive findings on both diffusion MRI and PET/CT [6]. Both methods show significant changes of parameters between active and inactive state of lymphomas, so, owing to its safety compared to PET/CT with its subsequent marked ionizing radiation, DW-MRI can be a valuable alternative for initial assessment of lymphoma and its advantages are enhanced especially when multiple follow-up scans are necessary.

The ADC maps are usually calculated using three $b$ values, with the first being close to 0 and the third being a multiple of second value. In our case we used b values of 50,400 and $800 \mathrm{~s} / \mathrm{mm}^{2}$. The $b$ value is a factor that determines how much effect fast or slow diffusion in tissues has on signal intensity. The relation between signal intensity and $b$ value is reflected by the Stejskal-Tanner formula (Eq. 1): 
2017. SIGNAL INTENSITY DYNAMICS OF MALIGNANT LYMPHOMA IN DIFFUSION-WEIGHTED MAGNETIC RESONANCE IMAGING...

$S=S_{o} e^{-b D}$,

where $S$ is the signal intensity after applying diffusion gradients, $S_{o}$ is the baseline signal intensity, $D$ is the apparent diffusion coefficient.

In conventional sequences, the effect of diffusion is miniscule. However, when multiple diffusion gradients are applied in DW imaging, these effects are amplified and become the main mechanism of tissue contrast [7]. Diffusion weighted images still contain contributions from spin densities and T1 and T2 relaxation times. Therefore, single diffusion weighted images can have a "T2 shine through" effect when a hyper intense lesion reflects strong T2 effect instead of reduced diffusion. By using more than one diffusion weighted image using different diffuse sensitization (b values), the effect of these factors can be eliminated and diffusion coefficient maps produced. $\mathrm{ADC}$ maps can be acquired with as few as two $\mathrm{b}$ values by calculating the apparent diffusion coefficient for each pixel:

$\mathrm{ADC}=\operatorname{In}\left[S_{2} / S_{1}\right] /\left(b_{1}-b_{2}\right)$,

where ADC - a measure of magnitude of diffusion, $S_{1}$ is the signal intensity when $b=b_{1}, S_{1} \neq$ $0 ; S_{2}$ is the signal intensity when $b=b_{2} ; b_{1}$ is the baseline $b$ value, usually close to $0, b_{1} \neq b_{2}$; $b_{2}$ is the second $\mathrm{b}$ value, $b_{2} \neq b_{1}$.

When using more $b$ values, the best fitting line for exponential decrease of signal intensity is calculated. However, it does not affect the line slope, which represents ADC and there in no change in its value [8]. After calculating ADC for each pixel, this data is visually presented as an ADC map. The coefficient is called apparent, because D value, or, in this case the ADC depends on experimental conditions. Average ADC values for a selected area in ADC map can be acquired.

Chemotherapy causes apoptosis of tumour cells, which shrink, leading to an increase in extracellular space, consequently increasing the value of ADC [9]. Later, as the tumour tissue reorganises and transforms into fibroid tissue, the ADC value drops. When analysing follow-up scans, in one case when tumour relapse was observed, the ADC value stayed elevated after 2 and 4 months post-treatment, so in this case the ADC value could be successfully used to predict relapse.

The use of DW-MRI in diagnosis of lymphoma picked up speed only several years ago and research concerning this matter is limited, however studies performed by other authors also showed comparable results. For illustration, Siegel et al. compared ADC values before treatment and after 2 courses of chemotherapy. Percentage changes in values for ADC were tested for differences from zero with the Wilcoxon signed-rank test. The medians and 25-75th percentile ranges were used for descriptive statistics. They proved that before treatment, average ADC value was $0,772 \times 10^{-3} \mathrm{~mm}^{2} / \mathrm{s}$, whereas after 2 courses of chemotherapy, the average statistically significantly increased by $85 \%$ to $1,561 \times 10^{-3} \mathrm{~mm}^{2} / \mathrm{s}$. Findings of DW-MRI and PET/CT were also compared. The results matched in $97 \%$ of cases, while Ann-Arbor stage matched in all cases. After 2 courses of chemotherapy 5 of 49 tumour sites remained abnormal on both DW-MRI and PET/CT, and there was one false positive on DW-MRI [10]. As in our case, a significant change of ADC was seen during treatment, while DW-MRI provided comparable results to those of $\mathrm{PET} / \mathrm{CT}$.

K. Tsuji et al. assessed the concordance of tumour stage between DW-MRI and PET/CT. Using MRI T2 sequences combined with diffusion imaging, the findings were same in $92,86 \%$ of cases. They also founded a significant difference in progress-free survival between patients with lesion positive and lesion negative MRI findings [11]. DW-MRI showed to be a valid alternative to PET/CT for differentiation of active and inactive lymphoma.

At the University of Pennsylvania Ming Q. Huang et al. performed a study aiming to evaluate the possible use of DW-MRI to detect response of human diffuse large $\beta$-cell lymphoma xenografts in severe combined immune deficient mice to chemotherapy. A significant change in both ADC value and T2 relaxation time were observed after one week of CHOPB chemotherapy 
[9]. These results suggest that DW-MRI can be used to evaluate the effectiveness of treatment at an early stage, allowing changing treatment strategy as soon as possible, avoiding the harmful side effects of/or ineffective treatment.

In our study, the change of ADC during the course of treatment could not be analysed due to lack of MRI scans performed mid-treatment, thus a prospective study would be valuable in determining the value of diffusion-weighted MRI for early assessment of treatment response in the future studies. However, a strong point of this study is the homogeneity of the cohort as lesion location and standard chemotherapy are the same for all patients and do not distort the results. Also the availability of follow up data, although currently limited to a couple of years, allowed to evaluate the dynamics of radiologic findings post-treatment.

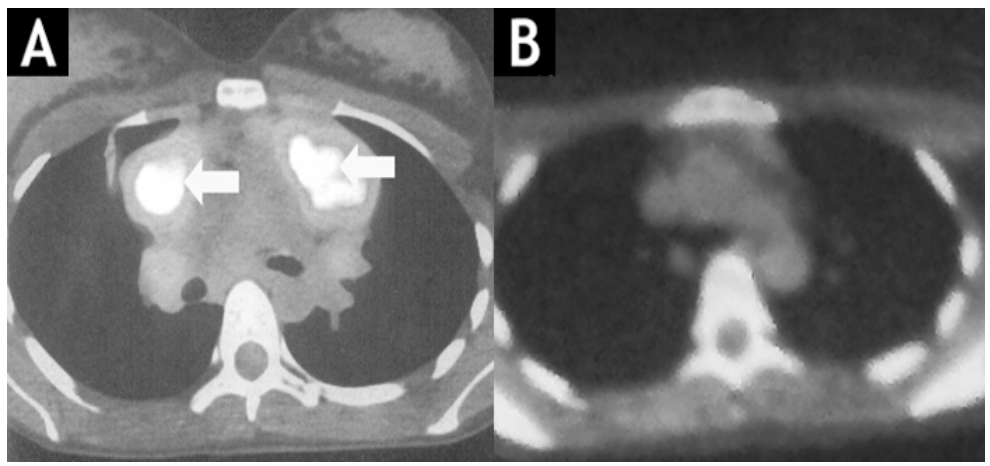

Fig. 1. A 26-year-old female patient with histological confirmed Hodgkin's lymphoma. PET/CT scan using [18] F-FDG radiopharmaceutical before (A) and after (B) treatment. Arrows show lymphoma masses with high FDG metabolism. No zones of high contrast accumulation were seen after treatment.

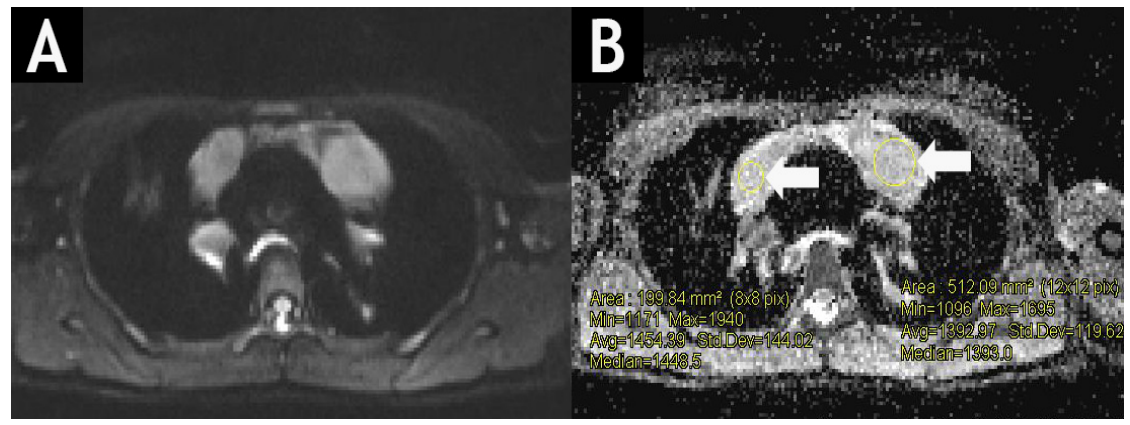

Fig. 2. Diffusion-weighted MRIT1WIBE (A) and ADC map (B) of the same patient. White arrows show active tumour masses of high signal intensity with an average ADC value of $1.42 \times 10^{-3} \mathrm{~mm}^{2} \mathrm{~s}$.

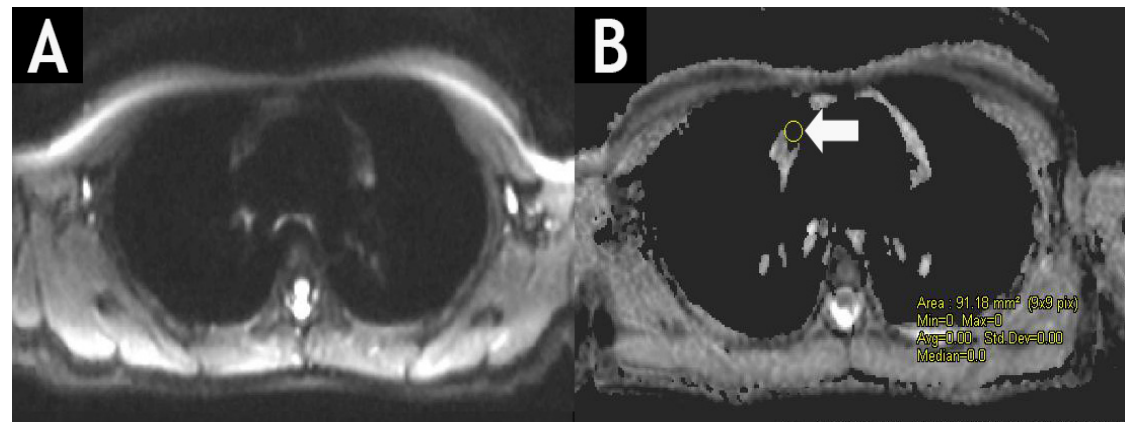

Fig. 3. DW-MRIT1WIBE (A) and ADC map (B) of the same patient after chemotherapy course. No masses of high signal intensity are observed in DW images with b values of 50,400 and $800 \mathrm{~s} / \mathrm{mm}^{2}$. In ADC map, the ADC value is lowering (immeasurable) in the former site of lymphoma masses. 
Algidas Basevicius - head of Radiology Department, LUHS Clinical Hospital; research coordinator. Laima Dobrovolskiene - collection and analysis of DW-MRI scans. Rytis Bliudzius - collection and analysis of PET/CT scans. Matas Juskevicius - selection of patients with lymphomas. Vincentas Veikutis - research coordination, statistical analysis. Vaidas Oberauskas - technical assistance, histology findings analysis.

\section{Conclusions}

We founded a statistically significant difference of values of ADC in active and inactive states of malignant lymphoma of mediastinal lymph nodes. Also statistically significant decrease of ADC value was observed after successful chemotherapy. Among the reviewed cases, DW-MRI had slightly worse specificity than PET/CT in differentiation of malignant and benign changes in lymph nodes, however further research of DW-MRI in diagnosis of malignant lymphomas should be carried out reviewing more cases to better compare the usefulness of these modalities.

\section{References}

[1] Ferlay J., Steliarova-Foucher E., Lortet-Tieulent J., Rosso S., Coebergh J. W. W., et al. Cancer incidence and mortality patterns in Europe: estimates for 40 countries in 2012. European Journal of Cancer, Vol. 49, 2013, p. 1374-1403.

[2] Xingchen Wu, Pirkko-Liisa Kellokumpu-Lehtinen. Diagnostic and Treatment Response Imaging in Lymphomas, Molecular Imaging. 2012.

[3] Xingchen Wu, Hannu Pertovaara, Pasi Korkola, Prasun Dastidar, Ritva Järvenpää, et al. Correlations between functional imaging markers derived from PET/CT and diffusion-weighted MRI in diffuse large B-cell lymphoma and follicular lymphoma. PLoS One, Vol. 9, Issue 1, 2014, p. 84999.

[4] Henzler T., Schmid-Bindert G., Schoenberg S. O., Fink C. Diffusion and perfusion MRI of the lung and mediastinum. European Journal of Radiology, Vol. 76, Issue 3, 2010, p. 329-36.

[5] Ciliberto M., Maggi F., Treglia G., Padovano F., Calandriello L., Giordano A., et al. Comparison between whole-body MRI and Fluorine-18-Fluorodeoxyglucose PET or PET/CT in oncology: a systematic review. Radiology and Oncology, Vol. 47, Issue 3, 2013, p. 206-218.

[6] Chieh Lin, et al. Whole-body diffusion magnetic resonance imaging in the assessment of lymphoma. Cancer Imaging, Vol. 12, Issue 2, 2012, p. 403-408.

[7] Kingsley P. B., Monahan W. G. Selection of the optimum $b$ factor for diffusion-weighted magnetic resonance imaging assessment of ischemic stroke. Magnetic Resonance in Medicine, Vol. 51, 2004, p. 996-1001.

[8] Michael Yong Park, Jae Young Byun Understanding the mathematics involved in calculating apparent diffusion coefficient maps. American Journal of Rentgenology, Vol. 199, 2012, p. 784-784.

[9] Huang M. Q., Pickup S., Nelson D. S., Qiao H., Xu H. N., Li L. Z., et al. Monitoring response to chemotherapy of non-Hodgkin's lymphoma xenografts by $\mathrm{T}(2)$-weighted and diffusion-weighted MRI. NMR in Biomedicine, Vol. 21, 2008, p. 1021-1029.

[10] Siegel M. J., Jokerst C. E., Rajderkar D., Hildebolt C. F., Goyal S., Dehdashti F., et al. Diffusion-weighted MRI for staging and evaluating response in diffuse large B-cell lymphoma: a pilot study. NMR in Biomedicine, Vol. 27, Issue 6, 2014, p. 681-691.

[11] Tsuji K., Kishi S., Tsuchida T., Yamauchi T., Ikegaya S., Urasaki Y., et al. Evaluation of staging and early response to chemotherapy with whole-body diffusion-weighted MRI in malignant lymphoma patients: a comparison with FDG-PET/CT. Journal of Magnetic Resonance Imaging, Vol. 41, Issue 6, 2015, p. 1601-1607.

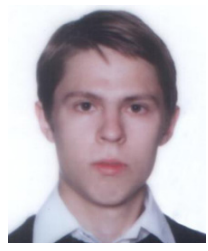

Rytis Bliudzius he is currently studying for a Master of medicine degree at the Medical Academy of Lithuanian University of Health Sciences. His research fields include diffusion-weighted imaging and positron emission tomography of malignant lymphoma, comparison of different radiologic modalities for evaluating the complexity of renal masses and nephrometry scoring in computed tomography. 
2017. SIGNAL INTENSITY DYNAMICS OF MALIGNANT LYMPHOMA IN DIFFUSION-WEIGHTED MAGNETIC RESONANCE IMAGING... RYTIS BLIŪDŽIUs, LAIMA DOBROVOLSKIENĖ, ALGIDAS BASEVIČIUS, VINCENTAS VEIKUTIS, MATAS JUŠKEVIČIUS, VAIDAS OBERAUSKAS

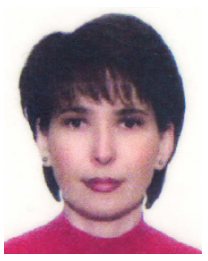

Laima Dobrovolskiene received the Ph.D. degree in biomedicine at Lithuanian University of Health Sciences. Now she is the Head of Sector of Thoracic Radiology at the Radiology Department of Lithuanian University Health Sciences hospital. Current research interests include comparing possibilities of different radiologic methods in diagnosis of recurrent malignant lymphomas of neck and body.

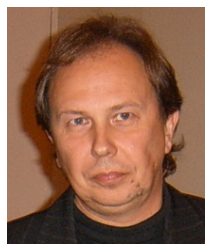

Algidas Basevicius physician radiologist, Professor Head of Radiology Department of LUHS, chairman of Kaunas Region and President of the Lithuanian Association of Radiologists. Currently his main field of interest is comparing different modalities and methods of quantifying the complexity of renal masses in radiologic diagnostics.

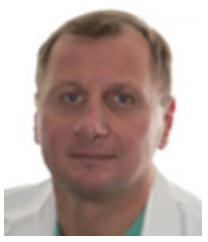

Vincentas Veikutis received M.D. degree in 1989 from Kaunas Medical Institute, Kaunas, Lithuania. Now he is Head of Laboratory of Electrophysiology and Experimental Surgery. Also he is Professor in Lithuanian University of Health Sciences. His current research activity includes experimental and clinical investigation in field of destructive energies (RF, US, laser) using and optimization efficacy. Next point of interest is thermography as field of radiology application in medicine clinical practice.

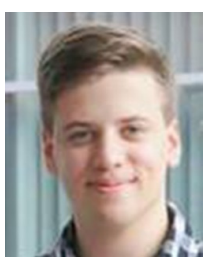

Matas Juskevičius is currently studying for a Master of medicine degree at the Medical Academy of Lithuanian University of Health Sciences. Currently his scientific work is aimed at comparing different radiologic methods in evaluation of malignant lymphoma masses. He is also investigating the value of diffenephrometry scores for initial assessment of renal tumours and predicting the outcome of treatment.

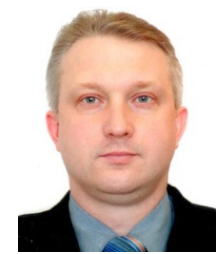

Vaidas Oberauskas received Ph.D. degree of Biomedical Sciences in Lithuanian Veterinary Academy 2004. Professor in Anatomy and Physiology department of LUHS. 